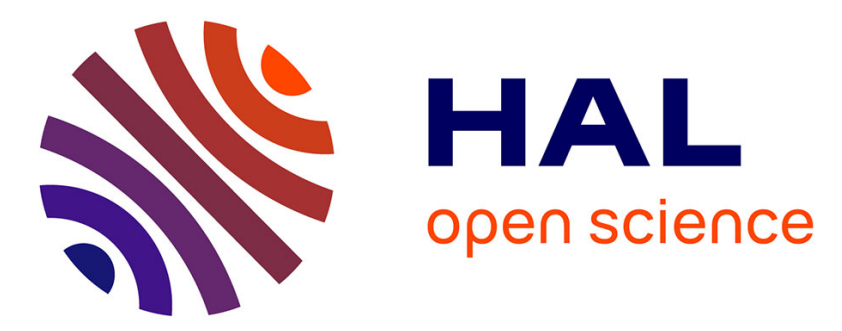

\title{
A Stochastic Multi-Temporal Optimal Power Flow Approach for the Management of Grid Connected Storage
}

Etta Grover-Silva, Xwégnon Ghislain Agoua, Robin Girard, Georges

Kariniotakis

\section{To cite this version:}

Etta Grover-Silva, Xwégnon Ghislain Agoua, Robin Girard, Georges Kariniotakis. A Stochastic MultiTemporal Optimal Power Flow Approach for the Management of Grid Connected Storage. CIRED 2017 (Congres International des Réseaux Electriques de Distribution), Jun 2017, Glasgow, United Kingdom. pp.12 - 15. hal-01500379

HAL Id: hal-01500379

https://hal-mines-paristech.archives-ouvertes.fr/hal-01500379

Submitted on 3 Apr 2017

HAL is a multi-disciplinary open access archive for the deposit and dissemination of scientific research documents, whether they are published or not. The documents may come from teaching and research institutions in France or abroad, or from public or private research centers.
L'archive ouverte pluridisciplinaire HAL, est destinée au dépôt et à la diffusion de documents scientifiques de niveau recherche, publiés ou non, émanant des établissements d'enseignement et de recherche français ou étrangers, des laboratoires publics ou privés. 


\title{
A STOCHASTIC MULTI-TEMPORAL OPTIMAL POWER FLOW APPROACH FOR THE MANAGEMENT OF GRID CONNECTED STORAGE
}

\author{
Etta GROVER-SILVA ${ }^{1,2} \quad$ Xwégnon Ghislain AGOUA ${ }^{1} \quad$ Robin GIRARD $^{1} \quad$ Georges KARINOTAKIS $^{1}$ \\ ${ }^{1}$ MINES ParisTech, PSL - Research University, PERSEE - Centre for Process Energies and Energy Systems France \\ ${ }^{2}$ ADEME - French Environment and Energy Management Agency - France \\ E-mail addresses: \{etta.grover-silva, xwegnon.agoua, robin.girard, georges.kariniotakis\}@mines-paristech.fr
}

\begin{abstract}
Renewable energy (RE) integration into distribution grids is becoming more common in the context of the energy transition. The management of wind or solar generation due to their variability and low predictability are challenging for distribution system operators (DSO). To that may be added uncertainties related to electric load profiles. The role of flexibility, coming from decentralized storage devices, will be important for DSOs trying to manage uncertain loads as well as high levels of $R E$ penetration. The introduction of automation and smart metering in distribution grids allows for the optimized management of storage devices to maximize the capability of current infrastructure to integrate $R E$ generators. These optimized management strategies can be calculated with optimal power flow (OPF) algorithms. This paper uses a convex relaxation of the power flow equations to expand the multi-temporal deterministic approach presented in [1] to a stochastic one. The stochastic algorithm implies the integration of a scenario tree to plan the charging and discharging schedule of batteries one day in advance. When comparing deterministic and stochastic operation planning strategies, the stochastic method annually increases total economic benefit by $3.1 \%$ while requiring lower annual cycling of the battery therefore increasing battery life.
\end{abstract}

\section{INTRODUCTION}

Renewable energy generators are becoming more common in the distribution system as a result of the transition from traditional electric generation to more sustainable technologies. These decentralized generators, such as photovoltaic (PV) systems, introduce new technical difficulties for distribution system operators (DSO) including bi-directional power flow, over current and voltage profile shifting. Smart meters are gaining popularity as a solution to improved visibility and controllability in distribution grids. The automation of distribution grids can allow for the optimization of existing grid architecture to avoid unnecessary infrastructure upgrades.

The new possibility to control decentralized generators and loads in the distribution system requires new and innovative management strategies. Optimal power flow algorithms are effective at calculating optimal set points for decentralized controllable devices while guaranteeing that no power quality requirements are violated. These types of algorithms can take into account the voltage and current constraints of an electrical distribution grid while minimizing an overall cost function.

At the distribution grid level, the uncertainties of renewable energy production and electric loads are high due to minimal aggregation effects. These uncertainties introduce a challenge for DSOs to optimize their controllable devices. Management strategies have been employed by DSOs to optimize decentralized controllable devices under uncertainty. These management strategies include real time flexibility of the system through grid connected storage or PV curtailment strategies managed by a real time distribution management system (DMS). However, real time control of a distribution system can imply sophisticated communication networks. This flexibility can also be scheduled on a day-ahead basis to reduce the need for real time power flow control.

Stochastic optimization has been presented in the literature as effective for planning operational strategies of controllable grid connected devices under uncertainty. For example reactive power compensation under uncertainty was explored by using statistical probability distribution curves and a Monte Carlo sampling technique to represent prediction errors in an OPF algorithm [2]. A similar probability distribution using multi-stage stochastic programming with a chanceconstrained optimization problem uses probabilistic penalty constraints associated with prediction errors [3]. However, these techniques imply a precise estimation of the distribution profile of errors associated with each forecast.

The use of scenarios to take uncertainties into account requires less detailed forecast data. Accounting for uncertainties through scenarios is present in the literature. For example, a simulation of a deterministic scenario and associated forecast limits of load and PV for a threephase distribution grid is presented in [4]. This analysis is a single time step analysis performed for each hour of the day. This daily analysis does not take into account variables that have temporal dependencies. A multitemporal algorithm is necessary to consider time 
dependent variables such as storage devices and controllable loads.

Multi-temporal stochastic OPF can be applied to distribution grids to analyze the benefits of grid connected storage devices under uncertainty. However, the integration of multi-temporal and multi-scenario aspects into an OPF algorithm can increase exponentially the size of the problem. Therefore, intelligent scenario selection and a certain decoupling are necessary to effectively resolve this type of problem for annual analysis. There exist few algorithms that take into account temporal dependencies and uncertainties at the same time for the distribution grid.

This paper presents a stochastic OPF algorithm whose structure allows the integration of scenarios and multitemporal aspects for day ahead planning of storage devices. It utilizes convex relaxations of the power flow equations in the form of a second order cone program (SOCP) and iterative cuts to guarantee exactness as described in [5]. Just as the convex relaxations exploit the radial geometry of distribution grids, a radial geometry is also used for the scenario tree. This innovative approach proposes a simple scenario generation strategy based on historical data that shows a higher performance in comparison to a deterministic day ahead planning strategy.

The paper organization includes the definition of the algorithm in section 2, the algorithmic performance in section 3, the demonstration of such an algorithm for a French case study in section 4 followed by the case study results in section 5 and final conclusions in section 6 .

\section{STOCHASTIC OPTIMAL POWER FLOW ALGORITHM}

\section{SOCP Optimization Problem Formulation}

The SOCP optimization objective function is defined in eq. (1).

$$
\begin{aligned}
& \min \cdot \sum_{t=0}^{T} C_{e, t}\left|P_{0, t}\right|+\sum_{t=0}^{T} \sum_{n=0}^{N} C_{e, t}\left[r_{i j} \boldsymbol{\ell}_{i j, t}+\right. \\
& \left.\left(\bar{P}_{p v, j, t}-P_{p v, j, t}\right)\right]
\end{aligned}
$$

Where the definition of node specific variables will use the convention below:

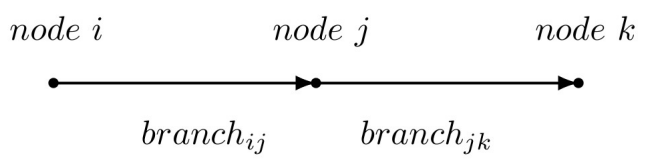

$C_{e, t}$ is the price of electricity for the time step $t, P_{0, t}$ is the active power at the substation at time step $t, r_{i j}$ is the resistance of branch $i j, \ell_{i j, t}$ is the square of the magnitude of the current on branch $i j$ at time $t, \bar{P}_{p v, j, t}$ is the maximum PV injection at node $j$ at time step $t, P_{l o s s, j, t}$ is the actual injection at node $j$ at time step $t$, and $P_{l o s s, j, t}$ is the losses of the battery system at node $j$ at time step $t$.

The optimization constraints include the PV power limits eq. (2), PV apparent power limits eq. (3) - eq. (4), the power flow equations eq. (5) - eq. (7), the relaxation of the current equation eq. (8) - eq. (9), the voltage limits eq. (10), battery power constraints eq. (11) and the constraints associated with the battery capacity eq. (12) eq. (13).

$$
\begin{aligned}
& P_{p v, j, t} \leq \bar{P}_{p v, j, t} \\
& S_{p v, j, t} \leq \bar{S}_{p v, j} \\
& S_{p v, j, t}=\sqrt{P_{p v, j, t}^{2}+Q_{p v, j, t}^{2}} \\
& P_{i j, t}=P_{l, j, t}+\sum_{k=0}^{K} P_{j k, t}+r_{i j} \ell_{i j, t}+P_{p v, j, t}+P_{s t, j, t}(5) \\
& Q_{i j, t}=Q_{l, j, t}+\sum_{k=0}^{K} Q_{j k, t}+x_{i j} \ell_{i j, t}+Q_{p v, j, t} \\
& v_{j, t}=v_{i, t}-2\left(r_{i j} P_{i j, t}+x_{i j} Q_{i j, t}\right)+\left(r_{i j}^{2}+x_{i j}^{2}\right) \boldsymbol{\ell}_{i j, t}(7) \\
& S_{i j, t} \geq \sqrt{P_{i j, t}^{2}+Q_{i j, t}^{2}} \\
& S_{i j, t} \geq \boldsymbol{\ell}_{i j, t} v_{i, t} \\
& \underline{V}^{2} \leq v_{j, t} \leq \bar{V}^{2} \\
& 0 \leq P_{s t, j} \leq \bar{P}_{s t, j} \\
& s o c_{s t, j, t-1, s}=s o c_{s t, j, t, s}+t P_{s t, j, t, s}+t P_{l o s s, j, t, s} \\
& P_{l o s s, j, t}=\eta \mid P_{s t, j, t}
\end{aligned}
$$

where $S_{p v, j, t}$ is the apparent power injection of the PV system at time step $t, \bar{S}_{p v, j}$ is the maximum apparent power rating of the $\mathrm{PV}$ inverter, $Q_{p v, j, t}$ is the apparent power of the PV system at time step $t, P_{i j, t}$ is the active power of branch $i j$ at time step $t, P_{l, j, t}$ is the active load at node $j$ at time step $t, P_{s t, j, t}$ is the active power injection of the battery system at node $j$ at time step $t, Q_{i j, t}$ is the reactive power of branch $i j$ at time step $t, Q_{l, j, t}$ is the reactive load at node $j$ at time step $t, x_{i j}$ is the reactance of branch $i j, Q_{p v, j, t}$ is the reactive power injection of the PV system at node $j$ at time step $t, v_{j, t}$ is the square of the voltage magnitude at node $\mathrm{j}$ at time step $t, \bar{V}$ is the maximum voltage, $\underline{V}$ is the minimum voltage, $s o c_{s t, j, t, s}$ is the state of charge of the battery system at node $j$ at time step $t$ for scenario $s$. The temporal and scenario dependencies are a result of eq. (12). 


\section{Scenario Generation and Structure}

Scenarios are generated for the PV production and load profiles for each node. The scenarios are generated by using historical PV production and load data. A quantile regression is completed using a historical learning set period of 60 days to then predict the $25^{\text {th }}, 50^{\text {th }}$ and $75^{\text {th }}$ quantile of PV production and load profile for the next two day period. The deterministic approach analyzes a daily period consisting of 24 coupled time steps and using the $50^{\text {th }}$ percentile for the PV production and load profiles for the predicted profiles. The stochastic approach combines the $25^{\text {th }}$ and $75^{\text {th }}$ quantile scenarios of PV and load profiles to create 4 combination scenarios. These combination scenarios are then used in conjunction with the $50^{\text {th }}$ quantile scenario to generate four stochastic OPF problems, here after called calculation blocks. Within each of these four calculation blocks there is a target period and a scenario period as shown by the time steps in black in Figure 2. The target period is defined as a period where there is only one variable representing the power of each battery system or controllable load which also corresponds to the $50^{\text {th }} \mathrm{PV}$ quantile and $50^{\text {th }}$ load quantile in all scenarios. The scenario period is characterized by multiple optimal values of the battery power injection or controllable load power for each time step. The physical meaning of multiple optimal values of the controllable power injection is the optimal power injection for each unique scenario. The target is coupled with each independent scenario therefore finding the optimal injection of the target period for all following injection possibilities. These blocks include using the $50^{\mathrm{th}}$ quantile for the target period and the 4 combination scenarios for the scenario period. The target period for the first three blocks is 6 hours while the target period for the last block is 12 hours. The deterministic problem scenario tree is found in Figure 1 while the stochastic OPF scenario tree can be found in Figure 2.

$$
\text { - } 50^{\text {th }} \text { PV, } 50^{\text {th }} \text { Load }
$$

$\begin{array}{lllllllllllllllllllll}0 & 1 & 2 & 3 & 4 & 5 & 6 & 7 & 8 & 9 & 10 & 11 & 12 & 13 & 14 & 15 & 16 & 17 & 18 & 19 & 20 \\ 21 & 22 & 23\end{array}$

Figure 1: Deterministic analysis with a single scenario using the $50^{\text {th }}$ quantile of $\mathrm{PV}$ and load profiles

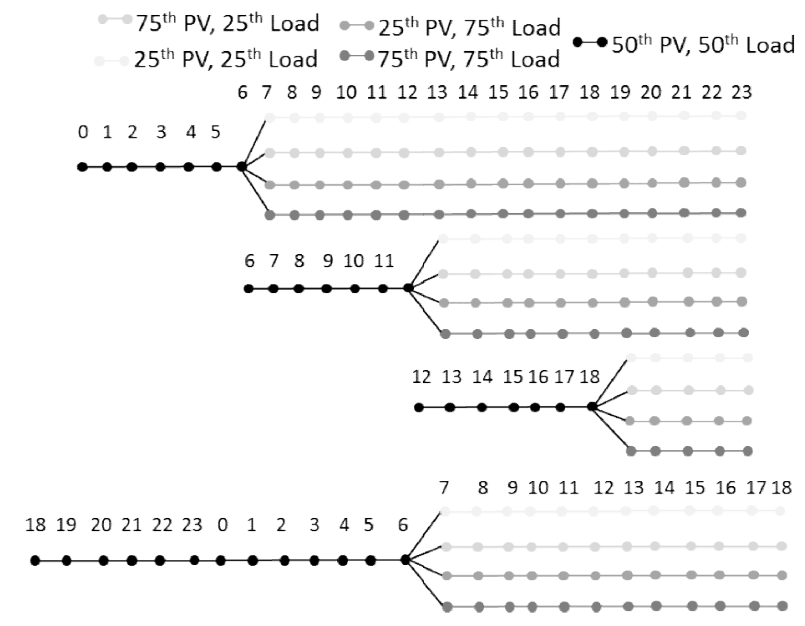

Figure 2: Stochastic analysis with a four dimensional scenario tree using $25^{\text {th }}, 5^{\text {th }}$ and $75^{\text {th }}$ quantiles of $P V$ and load profiles

After a scenario tree splits, the four combination scenarios are considered to be independent. Therefore the final optimal charging schedule of the battery is composed of the solution calculated during the target period for each block. For the deterministic problem, the target period is the same length as the total simulation period. Therefore only one problem is solved during the deterministic algorithm. The stochastic algorithm solves four different stochastic problems taking into account critical periods where scenarios could be significantly different. The coupling between the four blocks is done by setting the state of charge and power of the battery systems at the end of one target period equal to the first time step of the next block's target period. The final daily optimal charging schedule is composed of the target periods of each block.

\section{ALGORITHMIC PERFORMANCE}

An annual analysis is performed to calculate the annual benefit possible from using a stochastic day ahead scheduling in comparison to the deterministic day ahead scheduling. The algorithmic performance of the deterministic approach and the stochastic approach are shown in

\begin{tabular}{|l|c|c|}
\hline Approach & Coupled time steps & Time (s) \\
\hline Deterministic & 24 & 3.4 \\
\hline Stochastic & 24 & 31.1 \\
\hline
\end{tabular}

The deterministic algorithm has a lower calculation time in comparison to the stochastic algorithm.

\section{CASE STUDY}

\section{Grid Feeder}


An example urban electric grid in France was studied to demonstrate the use of the algorithm. The medium voltage distribution grid feeder is composed of 137 nodes with a nominal voltage of $30 \mathrm{kV}$ and is assumed to be located in Nice, France. A map of the grid topology can be found in Figure 3.

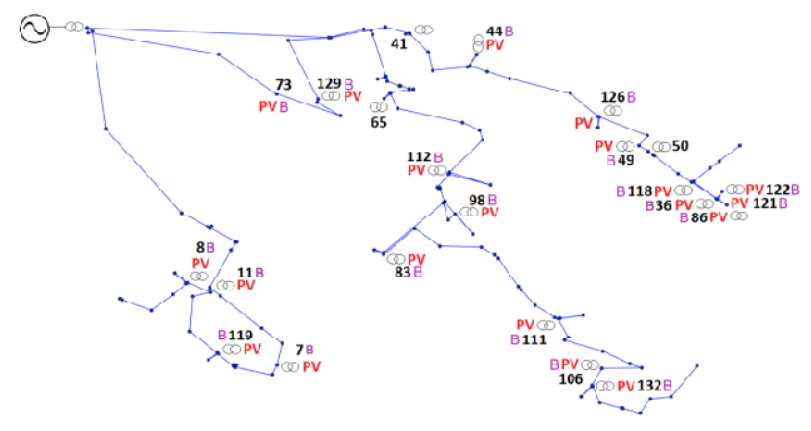

Figure 3: Grid topology with low voltage substation connections of medium voltage urban feeder in

\section{France.}

\section{$\underline{\text { Generation and Load Profiles }}$}

Electric load profile data was simulated with a bottom up load simulator detailed in [6] for each low voltage substation. A statistically accurate representation of residential and commercial customer proportion, electric heating, living surface area and population was simulated using the INSEE building inventory database of France. The location of each load profile was determined randomly due to the fact that no grid load data was available. The transformer connecting the medium voltage feeder and the high voltage grid is an $8 \mathrm{MVA}$ transformer serving 21 low voltage substations. Load profiles aligning with meteorological data in Grenoble, France indicated a peak load of 3.0 MW during the summer and 7.7 MW during the winter with an average load of respectively 2.8 MW and 4.2 MW. Solar system production data was based on the normalized real production of a PV plant in Grenoble France [7].

An amount of 20 PV systems and 20 battery systems were assigned to 20 nodes, a majority were chosen to be the same nodes as the nodes hosting low voltage substations. The size of these systems was chosen randomly to be between $35-250 \mathrm{~kW}$. Characteristics of the final load profile nodes can be found in Figure 4 and PV size information can be found in Figure 5.

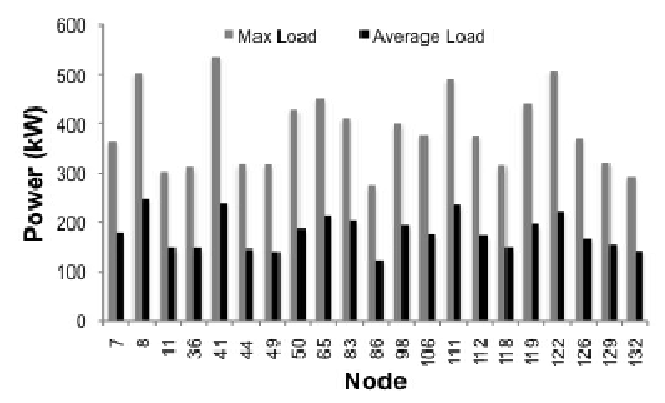

Figure 4: Maximum and average load for indicated

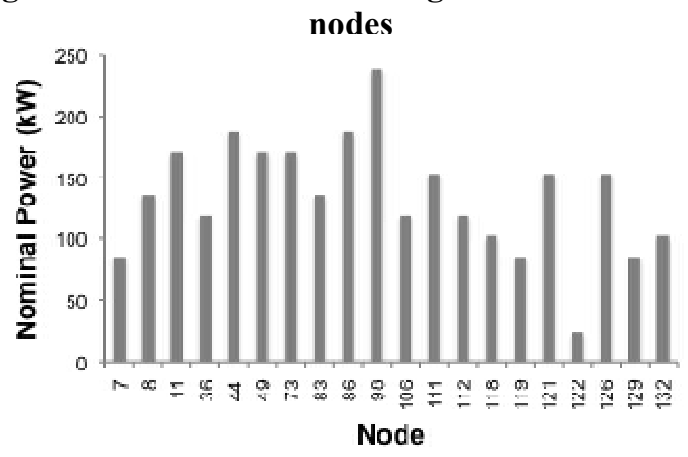

Figure 5: Nominal power of PV systems installed at indicated nodes

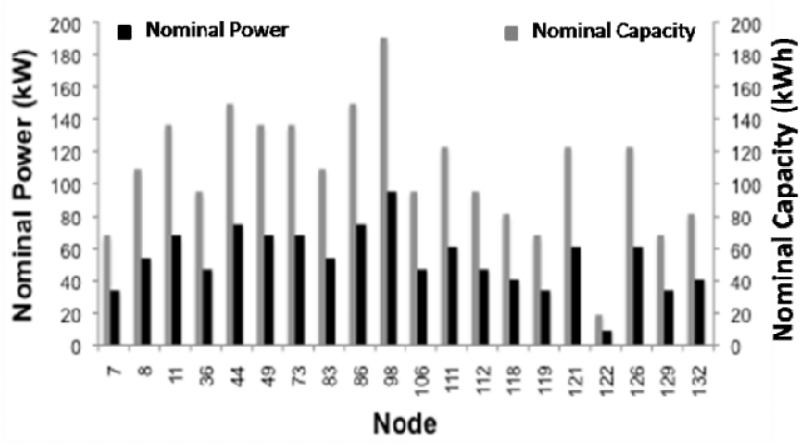

Figure 6: Nominal power and capacity of battery systems installed at indicated nodes

\section{RESULTS}

An annual analysis was performed to compare the effectiveness of day ahead planning for storage devices with stochastic and deterministic techniques. An example detailed profiles of the charging and discharging schedule for node 86 can be found in Figure 7. These profiles show the different charging profiles solved by the stochastic and deterministic algorithms.

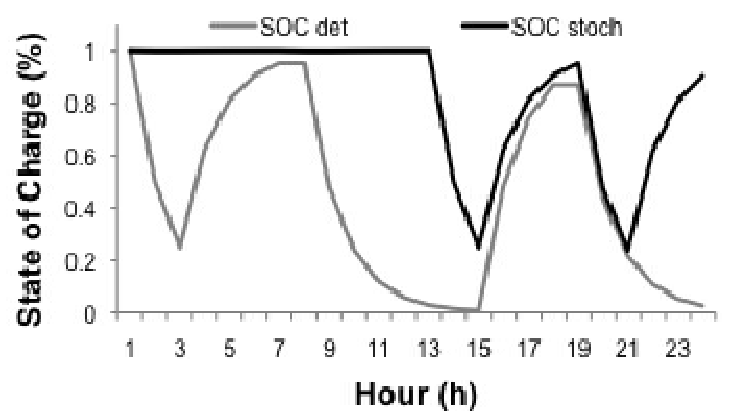




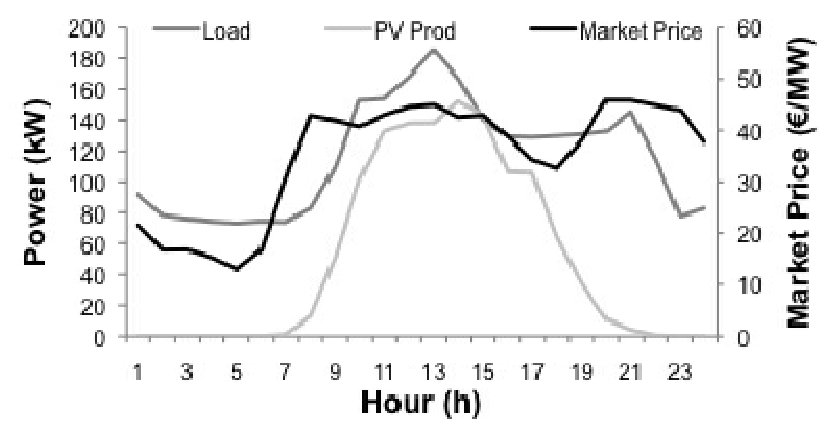

Figure 7: Charging schedule for July 11, 2012 of stochastic and deterministic planning methods (top) and associated load profile, PV production and market price (bottom)

As seen by the figure above, the stochastic battery charging schedule is more conservative using the battery less during the day. While the number of full cycles of the battery systems are lower in the stochastic case study the economic annual benefit is higher as seen in Table 1 .

This table shows that stochastic day ahead planning not only increases the battery life by decreasing the annual battery cycles but also increases annual economic benefit by $3.1 \%$.

Table 1: Comparison of stochastic and deterministic charging schedule annual performance

\begin{tabular}{|l|c|c|c|}
\hline Algorithm & $\begin{array}{c}\text { Energy } \\
(\mathrm{MWh})\end{array}$ & $\begin{array}{c}\text { Cost } \\
(\mathrm{M} €)\end{array}$ & $\begin{array}{c}\text { Battery } \\
\text { Cycles }\end{array}$ \\
\hline Deterministic & 30.02 & 1441 & 908 \\
\hline Stochastic & 30.65 & 1396 & 589 \\
\hline
\end{tabular}

\section{CONCLUSION}

This paper has presented two day-ahead battery operations scheduling for a distribution grid: one deterministic method and one stochastic method. These two methods are then compared quantifying the benefits of using stochastic analysis in distribution grid for storage operations management. The primary difference between the stochastic and deterministic analysis is that the stochastic algorithm calculated a more conservative use of the battery systems. This conservative usage decreases the number of cycles per year of the battery while increasing economic benefit by $3.1 \%$. Another advantage of this type of algorithm is that the quantile regression method used to calculate the scenarios has a low calculation burden and requires a short historical data set of only three months. Future work could be done on improving the scenario generation method to be more precise and integrating parallel programming to decrease the calculation time.
The authors would like to thank ENEDIS for the provision of some of the data used in this study. Mr. Thibaut Barbier is thanked for his support with the simulated load curves. This work is being carried out as a part of the research and innovation project SENSIBLE (Storage ENabled SustaInable energy for BuiLdings and communitiEs - www.h2020-projectsensible.eu) that has received funding from the European Union's under the Horizon 2020 Framework Programme (Grant No 645963). It is also supported from a $\mathrm{PhD}$ grant by ADEME (Agence de l'Environnement et de la Matrise de l'Energie) and ARMINES.

\section{REFERENCES}

[1] Florin Capitanescu, 2016, "Critical review of recent advances and further developments needed in $\mathrm{AC}$ optimal power flow", Electric Power Systems Research, vol. 136, 57-68.

[2] Vassilis Kekatos, Gang Wang, and Georgios B. Giannakis, 2014, "Stochastic Loss Minimization for Power Distribution Networks", Proceedings of North American Power Symposium, pp. 1-6

[3] T. Summers, J. Warrington, M. Morari and J. Lygeros, 2014, "Stochastic optimal power flow based on convex approximations of chance constraints", Proceedings of Power Systems Computation Conference, pp. 1-7

[4] M. Chehreghani Bozchalui, Chenrui Jin and R. Sharma, 2014, "Rolling Stochastic Optimization based operation of distribution systems with Pvs and Energy Storages", Proceedings of Innovative Smart Grid Technologies Conference (ISGT), IEEE PES, pp. 1-5

[5] S. Abdelouadoud, R. Girard, F. Neirac, and T. Guiot, 2015, "Optimal power flow of a distribution system based on increasingly tight cutting planes added to a second order cone relaxation", International Journal of Electrical Power \& Energy Systems, vol. 69, 9 17

[6] T. Barbier, R. Girard, F. P. Neirac, and G. Kariniotakis, 2014, "A novel approach for electric load curve holistic modeling and simulation", Proceedings of MedPower Conference, IET, pp $1-8$

[7] "Dix ans de production d'énergie solaire au Lycée du Grésivaudan de Meylan." Académie de Grenoble. November 28, 2011. http://www.acgrenoble.fr/admin/spip/spip.php?article3505

\section{ACKNOWLEDGEMENTS}

